Ana I. Pereira - Florbela P. Fernandes .

João P. Coelho · João P. Teixeira .

Maria F. Pacheco · Paulo Alves ·

Rui P. Lopes (Eds.)

Optimization, Learning

Algorithms and Applications

First International Conference, OL2A 2021

Bragança, Portugal, July 19-21, 2021

Revised Selected Papers

望 Springer 


\section{Editors}

Ana I. Pereira

Instituto Politécnico de Bragança

Bragança, Portugal

João P. Coelho

Instituto Politécnico de Bragança

Bragança, Portugal

Maria F. Pacheco

Instituto Politécnico de Bragança

Bragança, Portugal

Rui P. Lopes (D)

Instituto Politécnico de Bragança

Bragança, Portugal
Florbela P. Fernandes (D)

Instituto Politécnico de Bragança

Bragança, Portugal

João P. Teixeira (1D)

Instituto Politécnico de Bragança

Bragança, Portugal

Paulo Alves

Instituto Politécnico de Bragança

Bragança, Portugal

ISSN 1865-0929

ISSN 1865-0937 (electronic)

Communications in Computer and Information Science

ISBN 978-3-030-91884-2

ISBN 978-3-030-91885-9 (eBook)

https://doi.org/10.1007/978-3-030-91885-9

\section{(C) Springer Nature Switzerland AG 2021}

This work is subject to copyright. All rights are reserved by the Publisher, whether the whole or part of the material is concerned, specifically the rights of translation, reprinting, reuse of illustrations, recitation, broadcasting, reproduction on microfilms or in any other physical way, and transmission or information storage and retrieval, electronic adaptation, computer software, or by similar or dissimilar methodology now known or hereafter developed.

The use of general descriptive names, registered names, trademarks, service marks, etc. in this publication does not imply, even in the absence of a specific statement, that such names are exempt from the relevant protective laws and regulations and therefore free for general use.

The publisher, the authors and the editors are safe to assume that the advice and information in this book are believed to be true and accurate at the date of publication. Neither the publisher nor the authors or the editors give a warranty, expressed or implied, with respect to the material contained herein or for any errors or omissions that may have been made. The publisher remains neutral with regard to jurisdictional claims in published maps and institutional affiliations.

This Springer imprint is published by the registered company Springer Nature Switzerland AG The registered company address is: Gewerbestrasse 11, 6330 Cham, Switzerland 


\section{Preface}

The volume CCIS 1488 contains the refereed proceedings of the International Conference on Optimization, Learning Algorithms and Applications (OL2A 2021), an event that, due to the COVID-19 pandemic, was held online.

OL2A 2021 provided a space for the research community on optimization and learning to get together and share the latest developments, trends, and techniques as well as develop new paths and collaborations. OL2A 2021 had more than 400 participants in an online environment throughout the three days of the conference (July 19-21, 2021), discussing topics associated to areas such as optimization and learning and state-of-the-art applications related to multi-objective optimization, optimization for machine learning, robotics, health informatics, data analysis, optimization and learning under uncertainty, and the Fourth Industrial Revolution.

Four special sessions were organized under the following topics: Trends in Engineering Education, Optimization in Control Systems Design, Data Visualization and Virtual Reality, and Measurements with the Internet of Things. The event had 52 accepted papers, among which 39 were full papers. All papers were carefully reviewed and selected from 134 submissions. All the reviews were carefully carried out by a Scientific Committee of $61 \mathrm{PhD}$ researchers from 18 countries.

July 2021

Ana I. Pereira 


\section{Organization}

\section{General Chair}

Ana Isabel Pereira

Polytechnic Institute of Bragança, Portugal

\section{Organizing Committee Chairs}

Florbela P. Fernandes

João Paulo Coelho

João Paulo Teixeira

M. Fátima Pacheco

Paulo Alves

Rui Pedro Lopes

\section{Scientific Committee}

Ana Maria A. C. Rocha

Ana Paula Teixeira

André Pinz Borges

Andrej Košir

Arnaldo Cândido Júnior

Bruno Bispo

Carmen Galé

B. Rajesh Kanna

C. Sweetlin Hemalatha

Damir Vrančić

Daiva Petkeviciute

Diamantino Silva Freitas

Esteban Clua

Eric Rogers

Felipe Nascimento Martins

Gaukhar Muratova

Gediminas Daukšys

Glaucia Maria Bressan

Humberto Rocha

José Boaventura-Cunha

José Lima

Joseane Pontes

Juani Lopéz Redondo
Polytechnic Institute of Bragança, Portugal

Polytechnic Institute of Bragança, Portugal

Polytechnic Institute of Bragança, Portugal

Polytechnic Institute of Bragança, Portugal

Polytechnic Institute of Bragança, Portugal

Polytechnic Institute of Bragança, Portugal 


Jorge Ribeiro
José Ramos
Kristina Sutiene
Lidia Sánchez
Lino Costa
Luís Coelho
Luca Spalazzi
Manuel Castejón Limas
Marc Jungers
Maria do Rosário de Pinho
Marco Aurélio Wehrmeister
Mikulas Huba

Michał Podpora

Miguel Ángel Prada

Nicolae Cleju

Paulo Lopes dos Santos

Paulo Moura Oliveira

Pavel Pakshin

Pedro Luiz de Paula Filho

Pedro Miguel Rodrigues

Pedro Morais

Pedro Pinto

Rudolf Rabenstein

Sani Rutz da Silva

Sara Paiva

Sofia Rodrigues

Sławomir Stępień

Teresa Paula Perdicoulis

Toma Roncevic

Vitor Duarte dos Santos

Wojciech Paszke

Wojciech Giernacki
Polytechnic Institute of Viana do Castelo, Portugal

NOVA University Lisbon, Portugal

Kaunas University of Technology, Lithuania

University of León, Spain

University of Minho, Portugal

Polytecnhic Institute of Porto, Portugal

Marche Polytechnic University, Italy

University of León, Spain

Université de Lorraine, France

University of Porto, Portugal

Federal University of Technology - Paraná, Brazil

Slovak University of Technology in Bratislava, Slovakia

Opole University of Technology, Poland

University of León, Spain

Technical University of Iasi, Romania

University of Porto, Portugal

University of Trás-os-Montes and Alto Douro, Portugal

Nizhny Novgorod State Technical University, Russia

Federal University of Technology - Paraná, Brazil

Catholic University of Portugal, Portugal

Polytechnic Institute of Cávado e Ave, Portugal

Polytechnic Institute of Viana do Castelo, Portugal

Friedrich-Alexander-University of Erlangen-Nürnberg, Germany

Federal University of Technology - Paraná, Brazil

Polytechnic Institute of Viana do Castelo, Portugal

Polytechnic Institute of Viana do Castelo, Portugal

Poznan University of Technology, Poland

University of Trás-os-Montes and Alto Douro, Portugal

University of Split, Croatia

NOVA University Lisbon, Portugal

University of Zielona Gora, Poland

Poznan University of Technology, Poland 
Optimizing Data Transmission in a Wireless Sensor Network Based on LoRaWAN Protocol

Thadeu Brito, Matheus Zorawski, João Mendes,

Beatriz Flamia Azevedo, Ana I. Pereira, José Lima, and Paulo Costa

Indoor Location Estimation Based on Diffused Beacon Network . . . . . . . . . 294 André Mendes and Miguel Diaz-Cacho

SMACovid-19 - Autonomous Monitoring System for Covid-19 . . . . . . . . 309 Rui Fernandes and José Barbosa

\section{Optimization in Control Systems Design}

Economic Burden of Personal Protective Strategies for Dengue Disease:

an Optimal Control Approach

Artur M. C. Brito da Cruz and Helena Sofia Rodrigues

ERP Business Speed - A Measuring Framework ................. 336 Zornitsa Yordanova

BELBIC Based Step-Down Controller Design Using PSO

João Paulo Coelho, Manuel Braz-César, and José Gonçalves

Robotic Welding Optimization Using A* Parallel Path Planning Tiago Couto, Pedro Costa, Pedro Malaca, Daniel Marques, and Pedro Tavares

\section{Deep Learning}

Leaf-Based Species Recognition Using Convolutional Neural Networks Willian Oliveira Pires, Ricardo Corso Fernandes Jr., Pedro Luiz de Paula Filho, Arnaldo Candido Junior, and João Paulo Teixeira

Deep Learning Recognition of a Large Number of Pollen Grain Types . . . . . 381 Fernando C. Monteiro, Cristina M. Pinto, and José Rufino

Predicting Canine Hip Dysplasia in X-Ray Images Using Deep Learning . . . . . . Daniel Adorno Gomes, Maria Sofia Alves-Pimenta, Mário Ginja, and Vitor Filipe

Convergence of the Reinforcement Learning Mechanism Applied to the Channel Detection Sequence Problem 


\title{
Deep Learning Recognition of a Large Number of Pollen Grain Types
}

\author{
Fernando C. Monteiro ${ }^{10000-0002-1421-8006]}$, Cristina M. Pinto, and José \\ Rufino ${ }^{1[0000-0002-1344-8264]}$ \\ Research Centre in Digitalization and Intelligent Robotics (CeDRI), Instituto \\ Politécnico de Bragança, Campus de Santa Apolónia, 5300-253 Bragança, Portugal \\ monteiro@ipb.pt, cristinapinto1995@hotmail.com, rufino@ipb.pt \\ https://cedri.ipb.pt
}

\begin{abstract}
Pollen in honey reflects its botanical origin and melissopalynology is used to identify origin, type and quantities of pollen grains of the botanical species visited by bees. Automatic pollen counting and classification can alleviate the problems of manual categorisation such as subjectivity and time constraints. Despite the efforts made during the last decades, the manual classification process is still predominant. One of the reasons for that is the small number of types usually used in previous studies. In this paper, we present a large study to automatically identify pollen grains using nine state-of-the-art CNN techniques applied to the recently published POLEN73S ${ }^{1}$ image dataset. We observe that existing published approaches used original images without study the possible biased recognition due to pollen's background colour or using preprocessing techniques. Our proposal manages to classify up to $97.4 \%$ of the samples from the dataset with 73 different types of pollen. This result, which surpasses previous attempts in number and difficulty of pollen types under consideration, is an important step towards fully automatic pollen recognition, even with a large number of pollen grain types.
\end{abstract}

Keywords: Pollen recognition - Convolutional neural network · Deep learning · Image segmentation.

\section{Introduction}

Food fraud has devastating consequences, particularly in the field of honey production, which the U.S. Pharmacopeia Fraud Database ${ }^{2}$ has classified as the third largest area of adulteration, only behind milk and olive oil. Our aim is to find solutions to help solving this problem and prevent its recurrence. The determination of the botanical origin can be used to label honey and the knowledge of the geographic origin is a factor that influences considerably the commercial value of the product and can be used for quality control and to avoid fraud [6].

\footnotetext{
${ }^{1}$ https://doi.org/10.6084/m9.figshare.12536573.v1

${ }^{2}$ https://decernis.com/solutions/food-fraud-database/
} 
Although demanding, pollen grain identification and certification are crucial tasks, accounting for a variety of questions like pollination or palaeobotany, but also for other fields of research, including crime scene investigation [13], allergology studies [7] as well as the botanical and geographical studies concerning origins of honey to prevent honey labelling fraud [15]. However, most of the pollen classification is a time consuming, laborious and a highly skilled work, visually done by human operators using microscopes, trying to identify differences and similarities between pollen grains. These differences are, frequently, imperceptible among pollen grains and may lead to identification errors.

Despite the efforts to develop approaches that allow the automatic identification of pollen grains [22,11], the discrimination of features performed by qualified experts is still predominant [4]. Many industries, including medical, pharmaceutical and honey marketing, depend on the accuracy of this manual classification process, which is reported to be around $67 \%$ [19]. A notorious paper [22] from 1996 published a brief summary of the state of the art until then and, more importantly, the demands and needs of palynology to elevate the field to a higher level, thus making it a more powerful and useful tool.

Pattern recognition from images has a long and successful history. In recent years, deep learning, and in particular Convolutional Neural Networks (CNNs), has become the dominant machine learning approach in the computer vision field, specifically, in image classification and recognition tasks. Since the number of annotated pollen images in the publicly available datasets is too small to train a CNN from scratch, transfer learning can be employed. In this paper we propose an automatic pollen recognition approach divided into three steps: initially, the regions which contain pollen are segmented from the background; then, the colour is preprocessed; finally, the pollen is recognized using deep learning.

Most object recognition algorithms focus on recognizing the visual patterns in the foreground region of the images. Some studies indicate that convolutional neural networks $(\mathrm{CNN})$ are biased towards textures [3], whereas another set of studies suggests shape bias for a classification task [2]. However, little attention has been given to analyze how the recognition process is influenced by the background information in the training process.

Considering that there are certain similarities between the layers of a trained artificial network and the recognition task in the human visual cortex, in this study, we hypothesize that if the collected images of a pollen have a unique background colour, different from all the other pollens, it may biases the recognition task, since the recognition could be based only in the background colour. In order to study such influence we trained the CNN with several datasets: one composed with original images, another composed with segmented images (where the background colour was eliminated), and with preprocessed images with histogram equalization and contrast limit adaptive histogram equalization (CLAHE) techniques.

The acquisition of images usually has some different sources resulting in images with different background, as shown in Fig.1 from the POLEN73S dataset [1] used in this study. Deep learning based pollen recognition methods focus 
on learning visual features to distinguish different pollen grains. We observed that existing published approaches used the entire image with the original background, in the training process. Background and foreground pixels in each image contribute with the same influence into the learning algorithms. As each pollen type has a different background from other types, when those trained networks are used to classify the pollens they may be biased by capture relevance from pollen's background which may result in biased recognition.
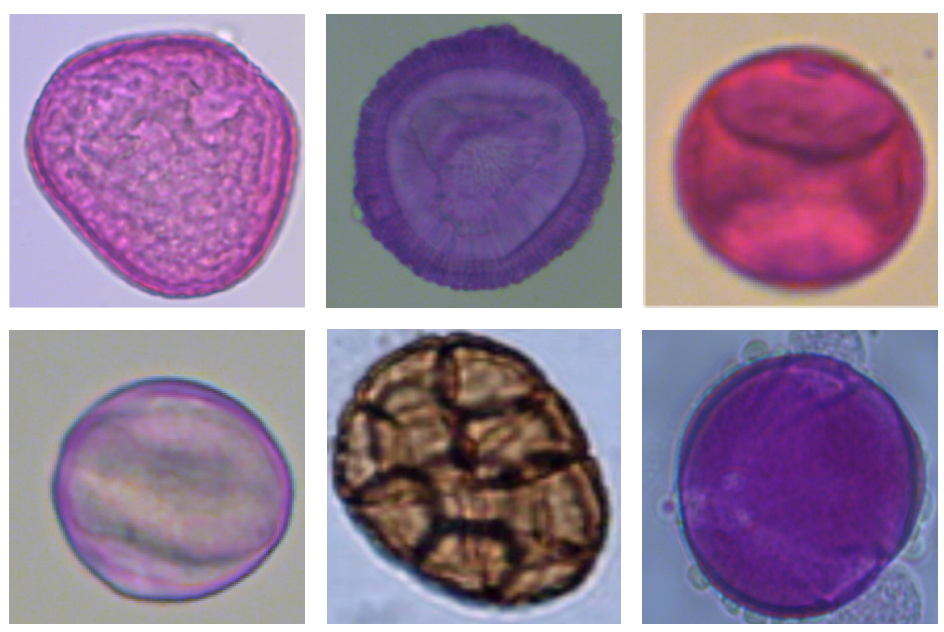

Fig. 1. Pollen dataset samples acquired with different background colours.

In this paper, we investigate the background and colour preprocessing influence by training nine state-of-the-art deep learning convolutional neural networks for pollen recognition. We used a recently published POLEN73S image dataset that includes more than three times as many pollen types and images as the POLEN23E dataset used in recent studies. Our approach manages to classify up to $97.4 \%$ of the samples from the dataset with 73 different types of pollen.

The remainder of this paper is organized as follows: Previous related works are presented and reviewed in Section 2. In Section 3, we describe the used materials and the proposed method. Section 4 presents the results and the discussion of the findings. Finally, some conclusions are drawn in Section 5.

\section{Related Works}

Automatic and semi-automatic systems for pollen recognition based on image features, in particular neural networks and support vector machines, have been proposed for a long time $[17,9,21,11]$. In general terms, those approaches extract some feature characteristics to identify each pollen type. 
Although the classification remains based on a combination of image features, the deep learning CNN approach builds a model determining and extracting the features itself, in alternative of being predefined by human experts. Several CNN learning techniques have been developed for classifying pollen grain images $[8,18,19,1]$. In [8], Daood et al. present an approach that learns from the image features and defines the model classifier from a deep learning neural network. This method achieved a $94 \%$ classification rate on a dataset of 30 pollen types. Sevillano and Aznarte in [18] and [19] proposed a pollen classification method that applied transfer learning on the POLEN23E dataset and to a 46 different pollen types dataset, achieving accuracies of over $95 \%$ and $98 \%$, respectively. In [1], Astolfi et al. presented the POLEN73S dataset and made an extensive study with several CNNs, achieving an accuracy of $95.8 \%$. Despite the importance of their study, we identify two drawbacks in their approach, that influenced the performance: they used different number of samples for each pollen type, and used, for each pollen, an image background that is different from the image background of other pollen types.

\section{$3 \quad$ Experimental Setup}

\subsection{Pollen Dataset}

The automation of pollen grain recognition depends on large image datasets with many samples categorized by palynologists. The results obtained depend on the number of pollen types and the number of samples used. Few samples may result in poor learning models, that are not sufficient to train conveniently the CNN; on the other hand, a small number of pollen types simplifies the identification process making it impractical to be used for recognizing large numbers of pollens usually found in a honey sample.

While a number of earlier datasets have been used for pollen grain classification, such as the POLEN23E ${ }^{3}$ dataset [9] or the Pollen Grain Classification Challenge dataset ${ }^{4}$, which contain 805 (23 pollinic types) and 11.279 (4 pollinic types) pollen images, respectively, in this paper we use POLLEN73S, which is one of the largest publicly available datasets in terms of pollen types number.

POLLEN73S is an annotated public image dataset, for the Brazilian Savannah pollen types. According to its description in [1] the dataset includes pollen grain images taken with a digital microscope at different angles and manually classified in 73 pollen types, containing 35 sample images for each pollen type, except gomphrena sp, trema micrantha and zea mays, with 10, 34 and 29 samples, respectively. From the results presented in [1], we observed that these small number of samples biased the results. Since CNNs were trained with a smaller number of samples for those types of pollens, this resulted in the worst classification scores relative to the other pollens. To overcome this problem, in our study, several images were generated through rotating and scaling the original images

\footnotetext{
${ }^{3}$ https://academictorrents.com/details/ee51ec7708b35b023caba4230c871ae1fa254ab3

${ }^{4}$ https://iplab.dmi.unict.it/pollenclassificationchallenge/
} 
of these pollen types, ensuring the same number of samples for each pollen type, which gives a total of 2555 pollen images. Although the images in the dataset have different width and height, they were resized accordingly with the image size of each CNN architecture input.

More datasets were constructed by removing the pollen's background colour (see Fig. 2). Since the images background has medium contrast with the pollen grains, the segmentation process uses just automatic thresholding and morphological operations. We also applied histogram equalization and contrast limit adaptive histogram equalization (CLAHE) to those segmented images. These new datasets allow the independence of training and testing processes from the background colour among the pollen types.
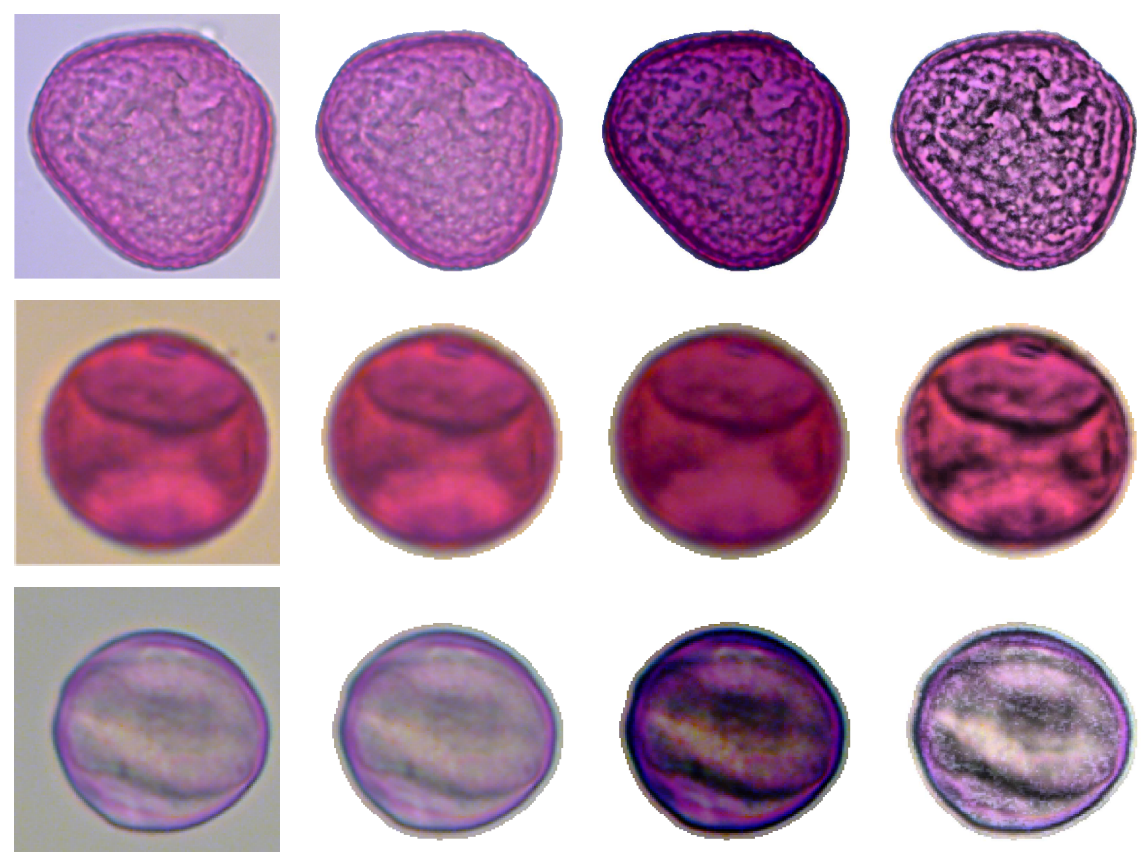

Fig. 2. First column: original images; second column: segmented images with background colour removed; third column: segmented equalized images; fourth column: segmented CLAHE images.

\subsection{Convolutional Neural Networks Architectures}

CNN is a type of deep learning model for processing images that is inspired by the organization of the human visual cortex and is designed to automatically create and learn feature hierarchies through back-propagation by using multiple layer blocks, such as convolution layers, pooling layers, and fully connected layers from low to high level patterns [2]. This technology is especially suited for image 
processing, as it makes use of hidden layers to convolve the features with the input data. The automatic extraction of the most discriminant features from a set of training images, suppressing the need for preliminary feature extraction, became the main strength of CNN approaches.

In this section, we present an overview of the main characteristics of the CNNs used in this study for the recognition of pollen grains types. We choose nine popular CNN architectures due to their performance on previous classification tasks. Table 1 contains a list (chronological sorted) of state-of-the-art CNN architectures, along with a high-level description of how the building blocks can be combined and how the information moves throughout the architecture.

\subsection{Transfer Learning}

Constraints of practical nature, such as the limited size of training data, degrade the performance of CNNs trained from scratch [18]. Since there is so much work that has already been done on image recognition and classification $[10,12,20]$, in this study we used transfer learning to solve our problem. With transfer learning, instead of starting the learning process from scratch, with a large number of samples, we can use previous patterns that have been learned when solving a similar classification problem.

Transfer learning is a technique whereby a CNN model is first trained on a large image dataset with a similar goal to the problem that is being solved. Several layers from the trained model, usually the lower layers, are then used in a new CNN, trained with sampled images from the current task. This way, the learned features in re-used layers are the starting points for the training process and adapted to classify new types of objects. Transfer learning has the benefit of reducing the training time for a $\mathrm{CNN}$ model and can overcome the generalization error due to the small number of images used in the training process when using a network from scratch.

The previous obtained weights, in each layer, may be used as the starting values for the next layers and adapted in response to the new problem. This usage treats transfer learning as a type of weight initialization scheme. This may be useful when the first related problem has a lot more labelled data than the problem of interest and the similarity in the structure of the problem may be useful in both contexts.

\subsection{Training process}

In the training process, the CNNs use the fine-tuning strategy, as well as the stochastic gradient descent with momentum optimizer (SGDM) at their default values, dropout rate set at 0.5 and early-stopping to prevent over-fitting, and the learning rate at 0.0001 . SGDM is used to accelerate gradients vectors in the correct directions, as we do not need to check all the training examples to have knowledge of the direction of decreasing slope, thus leading to faster converging. Additionally, to consume less memory and train the CNNs faster, we used the CNNs batch size at 12 to update the network weights more often, 
Table 1. Chronological list and descriptions of CNN architectures used in this paper.

VGG $16 / 19 \quad[20]$ Introduced the idea of using smaller filter kernels allowing for deeper networks, and training these networks using pretraining outputs of superficial layers. They have five convolutional blocks where the first two have convolution layers and one max-pooling layer in each block. The remaining three blocks have three fully-connected layers equipped with the rectification non-linearity (ReLU) and the final softmax layer.

ResNet 50 / 101 [10] Shares some design similarities with the VGG architectures. The batch normalization is used after each convolution layer and before activation. These architectures introduce the residual block that aims to solve the degradation problem observed during network training. In the residual block, the identity mapping is performed, creating the input for the next non-linear layer, from the output of the previous layer.

Inception-V3 [24] This network has three inception modules where the resulting output of each module is the concatenation of the outputs of three convolutional filters with different sizes. The goal of these modules is to capture different visual patterns of different sizes and approximate the optimal sparse structure. Finally, before the final softmax layer, an auxiliary classifier acts as a regularization layer.

Inception-ResNet [23] Uses the combination of residual connections and the Inception architecture. In Inception networks the gradient is backpropagated to earlier layers, and repeated multiplication may make the gradient indefinitely small, so they replaced filter concatenation stage with residual connections as in ResNet.

Xception

[5] The architecture is composed of three blocks, in a sequence, where convolution, batch normalization, ReLU, and max pooling operations are made. Besides, the residual connections between layers are made as in Resnet architecture.

DenseNet201

[12] Is based on the ideas of ResNet, but built from dense blocks and pooling operations, where each dense block is an iterative concatenation from all previous layers. In the main blocks, the layers are densely connected to each other. Massive reuse of residual information allows for deep supervision as each layer receives more information from the previous layer and therefore the loss function will react accordingly, which makes it a more powerful network.

DarkNet53 [16] It has 53 layers deep and acts as a backbone for the YOLOv3 object detection approach. This network uses successive convolutional layers with some shortcut connections (introduced by ResNet to help the activations propagate through deeper layers without gradient vanishing) to improve the learning ability. Batch Normalization is used to stabilize training, speed up convergence, and regularize the model batch. 
and trained them in 30 epochs. All images go through a heavy data augmentation which includes horizontal and vertical flipping, $360^{\circ}$ random rotation, rescaling factor between $70 \%$ and $130 \%$, and horizontal and vertical translations between -20 and +20 pixels. The CNNs were trained using the Matconvnet package for Matlab ${ }^{\circledR}$ on a node of the CeDRI cluster with two NVIDIA RTX 2080 Ti GPUs.

As in [1], we used 5-Set Cross-Validation, where in each set the images were split on two subsets, $70 \%$ (1825 images) for training and 30\% (730 images) for testing, allowing the CNN networks to be independently trained and tested on different sets. Since each testing set is build with images not seen by the training model, this allows us to anticipate the CNN behaviour against new images. The four datasets (original, segmented, segmented with equalization and segmented with CLAHE) were trained and tested in an independent way.

\section{Results and Discussion}

Other works use different evaluation metrics like Precision, Recall, F1-score [1] or correct classification rate (CCR) [18]. However, those metrics use the concept of true negative and false negative. As in this type of experiments we only obtain true positives or false positives, we evaluate the results with Accuracy (Precision gives the same score), which relates true positive with all possible results.

The evaluation results for the nine CNN architectures considered, with different colour pre-processing techniques, are presented in Table 2. The numbers exhibited in bold indicate the best Accuracy result obtained for each network.

Table 2. Classification results (in percentage) on the test set for the different CNNs and preprocessing techniques considered.

\begin{tabular}{lcccc}
\hline CNN & Original & Segmented & Seg. Equal. & Seg. CLAHE \\
\hline \hline VGG16 & 94.2 & $\mathbf{9 4 . 3}$ & 88.7 & 92.6 \\
\hline VGG19 & 91.9 & $\mathbf{9 2 . 4}$ & 92.1 & 92.1 \\
\hline ResNet50 & 95.1 & $\mathbf{9 5 . 2}$ & 93.2 & 94.8 \\
\hline ResNet101 & 94.5 & 94.6 & 93.0 & $\mathbf{9 5 . 2}$ \\
\hline Inception V3 & 92.3 & 92.8 & $\mathbf{9 4 . 0}$ & 90.8 \\
\hline Inception-ResNet & 92.3 & 89.9 & $\mathbf{9 2 . 4}$ & 90.3 \\
\hline Xception & $\mathbf{8 9 . 7}$ & 87.8 & 87.5 & 88.1 \\
\hline DenseNet201 & 96.7 & $\mathbf{9 7 . 4}$ & 96.6 & 95.9 \\
\hline DarkNet53 & 95.8 & $\mathbf{9 5 . 9}$ & 95.1 & 94.9 \\
\hline
\end{tabular}


Based on the results of Table 2, we can conclude that segmenting pollen grains images improves the classification performance for the majority of CNN models allowing the DenseNet201 to achieve an accuracy of $97.4 \%$. Only the Xception network produces better results for the original images. The Inception architectures achieve the best performance with segmented histogram equalized images. The remain architectures achieved the highest performance when using segmented images without any colour processing.

The DenseNet201 classified correctly all the images for 65 pollen grain types out the 73 types of the dataset. For the other 9 types, it misclassified up to two images, with a total of eleven false positives in the 730 tested images. The lowest accuracy result of the DenseNet201 was achieved with the pollen types dipteryx alata and myrcia guianensis. These pollen types have predominantly rounded shapes and high texture, that are normally learned in the first CNN layers. Since the transfer learning process changes only a set of the last CNN layers it does not change those learned features during the training process with our images, producing some misclassified results.

The accuracy rates achieved by the DenseNet201 network are relevant due to the amount of pollen types in the POLEN73S dataset, since Sevillano et al. [19] obtained a higher accuracy in a dataset containing only 46 pollen types. That shows that DenseNet201 presented an important performance on POLEN73S.

The network trained and tested using the segmented images produced false positives results that are misclassified as pollens that have high similarity with the tested ones. Figure 3 shows some of those false positive examples.
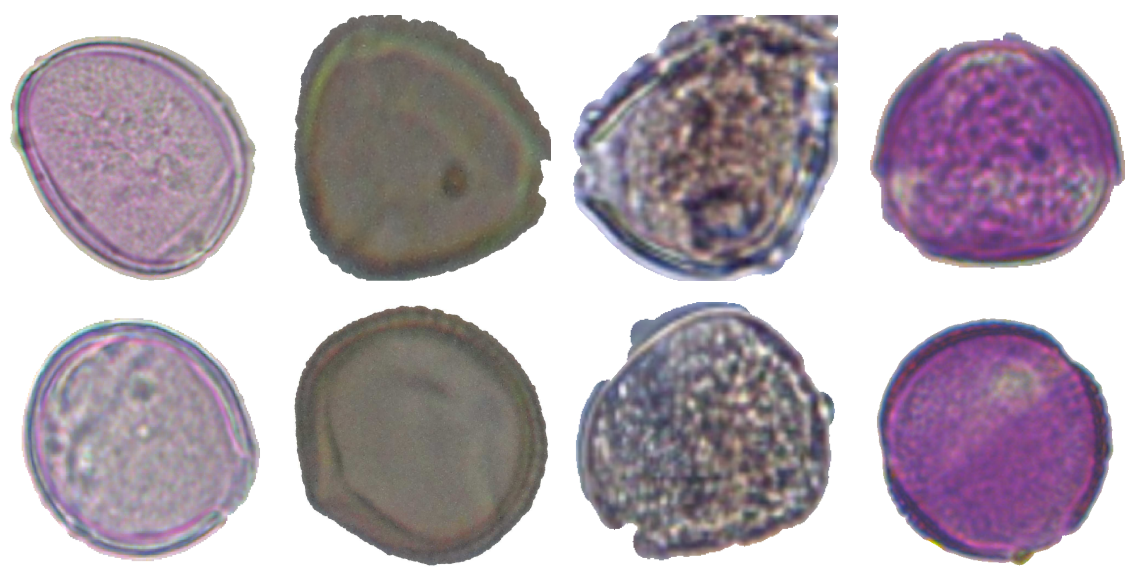

Fig. 3. First row: segmented tested pollens (magnolia champaca, myrcia guianensis, dipteryx alata, arachis sp); second row: misclassified pollens (ricinus communis, schizolobium parahyba, zea mays, myracroduon urundeuva). 
Table 3. Comparison with previous attempts at pollen classification of more than 20 pollen types using a CNN classifier, with number of types and the highest reported accuracy.

\begin{tabular}{lcc}
\hline Authors & \#Types & Accuracy \\
\hline \hline Sevillano et al. [18] & 23 & 97.2 \\
\hline Menad et al. [14] & 23 & 95.0 \\
\hline Daood et al. [8] & 30 & 94.0 \\
\hline Sevillano et al. [19] & 46 & 97.8 \\
\hline Astolfi et al. [1] & 73 & 95.8 \\
\hline Our approach & $\mathbf{7 3}$ & 97.4 \\
\hline
\end{tabular}

In networks trained and tested with segmented images the background colour bias information was removed, and so the pollen is classified using only the grain pollen information, correcting some of the false positives of the network trained with original images, where the background colour was used as a feature in the classification process.

The high values for the evaluation metric in all CNNs show that the number of correctly identified pollens is high when compared to the number of tested images. We believe that an accuracy over $97 \%$ is enough to build an automatic classification system of pollen grains, since the visual classification performed by human operators is a hard and time consuming task with a lower performance.

\subsection{Comparison with other studies}

We compared our results with other automatic approaches, from the current literature, that used a CNN classifier. Previous deep learning approaches have shown similar or higher accuracy rates to ours, but these studies were conducted with a small number of pollen types. Table 3 provides a summary table of previous studies, including class sizes and accuracy/success rates against our result. All the literature reviewed, except [1], used a significantly smaller image dataset, in terms of pollen types, than the one used in this paper.

Although the work of Sevillano et al. [19], with forty six types of pollen, achieved a slightly higher performance than our study, as the number of pollen types is directly related to the classification performance of the CNNs, the results must be evaluated taking into account this difference in the number of pollen types between the work presented in [19] and ours. 
In short, it can thus be concluded that training a network with the attention focused on the object itself by removing the background dissimilarities can improve the performance of CNN model in pollen classification problem.

\section{Conclusion}

The usual method for pollen grains identification is a qualitative approach, based on the discrimination of pollen grain characteristics by an human operator. Even though this manual method is quite effective, the all process is time consuming, laborious and sometimes subjective. Creating an automatic approach to identify the grains, in a precise way, thus represents a task of utmost interest.

In this study, an automated pollen grain recognition approach is proposed. We investigate the influence of background colours and colour pre-processing in the recognition task using nine state-of-the-art CNN topologies. Using a combination of an image-processing workflow and a sufficiently trained deep learning model, we were able to recognize pollen grains from seventy three pollen types, one of the largest number of pollen types studied until now, achieving an accuracy of $97.4 \%$ that represents one of the best success rate so far (when weighted for the number of pollen types used in this work).

This study proves that using deep learning CNN architectures for the pollen grain recognition task allows good classification results when using a transfer learning approach. In the future, we plan to combine the features from several CNNs enhancing the effectiveness of deep learning approaches in pollen grain recognition.

\section{References}

1. Astolfi, G., Gonçalves, A.B., Menezes, G.V., Borges, F.S.B., Astolfi, A.C., Matsubara, E., Alvarez, M., Pistori, H.: POLLEN73S: An image dataset for pollen grains classification. Ecological Informatics 60, 101165 (2020)

2. Baker, N., Lu, H., Erlikhman, G., Kellman, P.J.: Local features and global shape information in object classification by deep convolutional neural networks. Vision Research 172, 46-61 (2020)

3. Bianco, S., Cusano, C., Napoletano, P., Schettini, R.: Improving CNN-based texture classification by color balancing. Journal of Imaging 3(33) (2017)

4. Buters, J., Antunes, C., Galveias, A., Bergmann, K.C., Thibaudon, M., Galán, C., Schmidt-Weber, C., Oteros, J.: Pollen and spore monitoring in the world. Clinical and translational allergy $\mathbf{8}(9)(2018)$

5. Chollet, F.: Xception: Deep learning with depthwise separable convolutions. In: 2017 IEEE Conference on Computer Vision and Pattern Recognition. pp. 18001807 (2017)

6. Corvucci, F., Nobili, L., Melucci, D., Grillenzoni, F.V.: The discrimination of honey origin using melissopalynology and raman spectroscopy techniques coupled with multivariate analysis. Food Chemistry 169, 297-304 (2015)

7. D’Amato, G., Cecchi, L., Bonini, S., Nunes, C., Annesi-Maesano, I., Behrendt, H., Liccardi, G., Popov, T., van Cauwenberge, P.: Allergenic pollen and pollen allergy in Europe. Allergy 62(9), 976-990 (2007) 
8. Daood, A., Ribeiro, E., Bush, M.: Pollen grain recognition using deep learning. In: Advances in Visual Computing. pp. 321-330. Springer Int. Publishing (2016)

9. Gonçalves, A.B., Souza, J.S., Silva, G.G., Cereda, M.P., Pott, A., Naka, M.H., Pistori, H.: Feature extraction and machine learning for the classification of Brazilian savannah pollen grains. PLoS ONE 11(6), e0157044 (2016)

10. He, K., Zhang, X., Ren, S., Sun, J.: Deep residual learning for image recognition. In: 2016 IEEE Conference on Computer Vision and Pattern Recognition (CVPR). pp. $770-778$ (2016)

11. Holt, K.A., Bennet, K.D.: Principles and methods for automated palynology. New Phytologist 203(3), 735-742 (2014)

12. Huang, G., Liu, Z., Van Der Maaten, L., Weinberger, K.Q.: Densely connected convolutional networks. In: IEEE Conference on Computer Vision and Pattern Recognition (CVPR). pp. 2261-2269 (2017)

13. Laurence, A.R., Bryant, V.M.: Forensic Palynology, pp. 1741-1754. Springer New York, New York, NY (2014)

14. Menad, H., Ben-Naoum, F., Amine, A.: Deep convolutional neural network for pollen grains classification. JERI (2019)

15. Ponnuchamy, R., Bonhomme, V., Prasad, S., Das, L., Patel, P., Gaucherel, C., Pragasam, A., Anupama, K.: Honey pollen: Using melissopalynology to understand foraging preferences of bees in tropical south India. PLoS ONE 9(7), e101618 (2014)

16. Redmon, J., Farhadi, A.: YOLOv3: An incremental improvement. ArXiv p. 1804.02767 (2018)

17. Rodriguez-Damian, M., Cernadas, E., Formella, A., Fernandez-Delgado, M., Pilar De Sa-Otero: Automatic detection and classification of grains of pollen based on shape and texture. IEEE Transactions on Systems, Man, and Cybernetics, Part C (Applications and Reviews) 36(4), 531-542 (2006)

18. Sevillano, V., Aznarte, J.L.: Improving classification of pollen grain images of the POLEN23E dataset through three different applications of deep learning convolutional neural networks. PLoS ONE 13(9), e0201807 (2018)

19. Sevillano, V., Holt, K., Aznarte, J.L.: Precise automatic classification of 46 different pollen types with convolutional neural networks. PLoS ONE 15(6), e0229751 (2020)

20. Simonyan, K., Zisserman, A.: Very deep convolutional networks for large-scale image recognition. In: Int. Conference on Learning Representations. pp. 1-14 (2015)

21. Sobol, M.K., Finkelstein, S.A.: Predictive pollen-based biome modeling using machine learning. PLoS ONE 13(8), e0202214 (2018)

22. Stillman, E., Flenley, J.: The needs and prospects for automation in palynology. Quaternary Science Reviews 15(1), 1-5 (1996)

23. Szegedy, C., Ioffe, S., Vanhoucke, V., Alemi, A.: Inception-v4, inceptionresnet and the impact of residual connections on learning. In: Proceedings of the Thirty-First AAAI Conference on Artificial Intelligence. pp. 4278-4284 (2017)

24. Szegedy, C., Vanhoucke, V., Ioffe, S., Shlens, J., Wojna, Z.: Rethinking the inception architecture for computer vision. In: 2016 IEEE Conference on Computer Vision and Pattern Recognition (CVPR). pp. 2818-2826 (2016) 